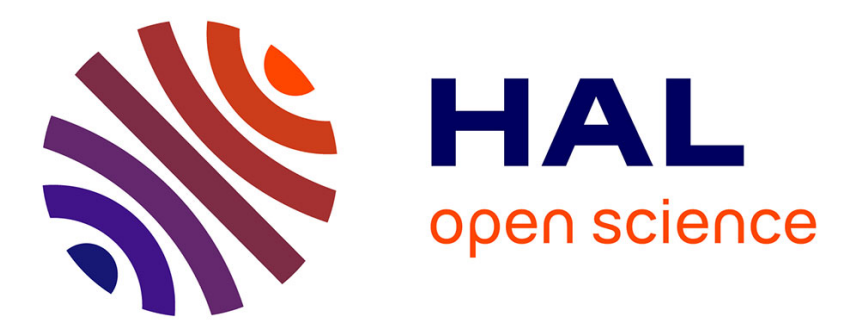

\title{
LES-SSAM for a high Reynolds number turbulent channel flow
}

Rémi Zamansky, Ivana Vinkovic, Mikhael Gorokhovski

\section{To cite this version:}

Rémi Zamansky, Ivana Vinkovic, Mikhael Gorokhovski. LES-SSAM for a high Reynolds number turbulent channel flow. QLES 2009, Sep 2009, Pise, Italy. pp.69-78, 10.1007/978-94-007-0231-8_6 . hal-00683695

\section{HAL Id: hal-00683695 https://hal.science/hal-00683695}

Submitted on 29 Mar 2012

HAL is a multi-disciplinary open access archive for the deposit and dissemination of scientific research documents, whether they are published or not. The documents may come from teaching and research institutions in France or abroad, or from public or private research centers.
L'archive ouverte pluridisciplinaire HAL, est destinée au dépôt et à la diffusion de documents scientifiques de niveau recherche, publiés ou non, émanant des établissements d'enseignement et de recherche français ou étrangers, des laboratoires publics ou privés. 


\title{
LES-SSAM for a high Reynolds number turbulent channel flow
}

\author{
Remi Zamansky, Ivana Vinkovic and Mikhael Gorokhovski \\ LMFA UMR 5509 CNRS Ecole Centrale de Lyon Université Claude Bernard \\ Lyon 1, 36 avenue Guy de Collongue, 69134 Ecully Cedex, France \\ remi.zamansky@ec-lyon.fr, ivana.vinkovic@ec-lyon.fr, \\ mikhael.gorokhovski@ec-lyon.fr
}

Summary. In [15] the large eddy simulation with stochastic modeling of the subgrid acceleration (LES-SSAM) for homogeneous turbulence was proposed. The main motivation of this approach is to account for intermittency of the flow at sub-grid scales, by emphasizing the role of sub-grid acceleration. In this paper, we develop further this approach in order to simulate a high Reynolds number channel flow. We proposed a new sub-grid acceleration model for channel flow. This model introduces explicitly the cross-channel correlation of subgrid velocity gradients and includes two parameters: the Reynolds number based on the friction velocity, and the channel half-width. The objective is to assess the capability of this model in comparison to the standard large-eddy simulation (LES) and to direct numerical simulation (DNS).

Key words: large-eddy simulation, sub-grid acceleration, stochastic model, intermittency, turbulent channel flow

\section{Introduction}

The structure of well-developed turbulent wall layer in the channel flow is highly intermittent. Close to the wall, the low-speed regions are interleaved with tiny zones of high-speed motion. The main role in this intermittency is attributed to quasi-streamwise vortices formed in the near-wall layer $[1,8,19]$.

Their anisotropic dynamics are Reynolds-number dependent. Sweeps from the outer layer toward the wall induce strong variations of the wall-normal velocity. The cross-channel correlation in the turbulent velocity field is amplified by merging of near-wall small-scale structures and their eruptions towards the outer region $[6,7,18]$.

For a high Reynolds flow, the LES at moderate resolution has to be combined with a SGS model for the non-resolved turbulent motion. The majority of SGS models are focused on simulation of turbulent stresses generated by the non-resolved velocity field $[3,10,14]$. In these models the structure of subgrid flow is supposed to be independent of the Reynolds number, i.e. to 
be not intermittent. Therefore the approach recently proposed by Sabelnikov [15] is focused directly on the stochastic modeling of the subgrid acceleration (LES-SSAM).

It was shown, by Kolmogorov's scaling, that, for a given filter width $\Delta$, the non-resolved acceleration may be substantially greater than the resolved acceleration: $\left(\bar{a}_{k} \bar{a}_{k}\right) /\left(a_{i}^{\prime} a_{i}^{\prime}\right) \approx(\eta / \Delta)^{2 / 3}$, where $\bar{a}_{k}$ and $a_{i}^{\prime}$ represent resolved and non-resolved accelerations and $\eta=L / R e_{L}^{3 / 4}$ is the Kolmogorov's length scale. This implies that in any SGS model, which is aimed to introduce the intermittency effects, the non-resolved acceleration must be a key variable. This motivated us to set up a new stochastic model for the subgrid acceleration of wall bounded flow. The aim of this paper is to assess the capability of the new model to reproduce the near-wall behavior compared to a standard LES and DNS.

\section{LES-SSAM approach and model formulation}

In the LES-SSAM framework of Sabelnikov [15], it is consider that the total instantaneous acceleration, governed by the Navier-Stokes equations, can be represented by the sum of two parts: $a_{i}=\bar{a}_{i}+a_{i}^{\prime}$. The first part represents the spatially filtered total acceleration: $\bar{a}_{i}=\frac{\partial \bar{u}_{i}}{\partial t}+\frac{\partial \bar{u}_{k} u_{i}}{\partial x_{k}}$, and is equivalent, with spatial filtering of the Navier-Stokes equations, to:

$$
\bar{a}_{i} \equiv \frac{\overline{d u_{i}}}{d t}=-\frac{1}{\rho} \frac{\partial \bar{p}}{\partial x_{i}}+\nu \Delta \bar{u}_{i} ; \quad \frac{\partial \bar{u}_{k}}{\partial x_{k}}=0
$$

with $\nu$ the kinematic viscosity. The second part is associated with the total acceleration in the residual field and is considered as a stochastic variable. In the LES-SSAM approach, eq. 1 is modeled in the framework of the classical LES approach. The resulting model-equation, which reconstructs an approximation for the non-filtered velocity field, writes then as:

$$
\frac{\partial \hat{u}_{i}}{\partial t}+\hat{u}_{k} \frac{\partial \hat{u}_{i}}{\partial x_{k}}=-\frac{1}{\rho} \frac{\partial \hat{p}}{\partial x_{i}}+\frac{\partial}{\partial x_{k}}\left(\nu+\nu_{t}\right)\left(\frac{\partial \hat{u}_{i}}{\partial x_{k}}+\frac{\partial \hat{u}_{k}}{\partial x_{i}}\right)+\hat{a}_{i}^{\prime} ; \quad \frac{\partial \hat{u}_{k}}{\partial x_{k}}=0
$$

where $\hat{\bullet}$ represents a synthetic field and $\nu_{\text {turb }}$ is given by an eddy viscosity model (e.g. the Smagorinsky subgrid model).

In [15] a model have been introduced for subgrid scale acceleration in isotropic and homogeneous turbulent flow. For further development of the LES-SSAM approach, we propose a new model for turbulent channel flow. We introduce the separation of variables for the non-resolved acceleration $\hat{a}_{i}^{\prime}$. On the basis of our DNS for turbulent channel flow (see table 1) and experiences $[9,11,12],|a|$, the modulus of the subgrid acceleration and $e_{i}$ its orientation, are two independent random variables, characterized by long memory and 
rapid decorrelation, respectively. Then the non-resolved acceleration is written as:

$$
\hat{a}_{i}^{\prime}=|a| e_{i}
$$

For $|a|$, our proposal is to emulate the modulus of the non-resolved acceleration in the following form:

$$
|a|=f \delta_{y} u_{*}^{2} / \nu
$$

where $\delta_{y}$ is the cell size in the normal to the wall direction and $u_{*}$ the friction velocity, $u_{*}^{2} /\left.\nu \equiv \frac{\partial u}{\partial y}\right|_{\text {wall }}$; so $\delta_{y} u_{*}^{2} / \nu$ will be considered as a typical normal to wall velocity increment in the near to wall region. $f$ is the subgrid frequency, considered as a stochastic variable. The frequency $f$ is supposed to have a stochastic evolution from the wall to the outer flow driven by the non-dimensional parameter $\tau$ defined as follows:

$$
\tau=-\ln \left(\frac{h-y}{h}\right)
$$

where $h$ is the channel half-width, and $y$ is the wall distance $(y=0: \tau=$ 0 and $y \rightarrow h: \tau \rightarrow \infty)$. The near-wall region is characterized by strong velocity gradients (high values of $f$ ), which decrease in mean toward the outer flow through the highly intermittent boundary layer. Thereby we assumed that with increasing of the normal distance from the wall, the frequency $f$ is changing by a random independent multiplier $\alpha(0<\alpha<1)$, governed by distribution $q(\alpha), \int_{0}^{1} q(\alpha) d \alpha=1$, which is in principle unknown. In other words, we apply the fragmentation stochastic process under scaling symmetry for the frequency $f$. From [4], we derive the following stochastic equation corresponding to this process:

$$
d f=\left(\langle\ln \alpha\rangle+\left\langle\ln ^{2} \alpha\right\rangle / 2\right) f d \tau+\sqrt{\left\langle\ln ^{2} \alpha\right\rangle / 2} f d W(\tau)
$$

where $\left\langle l n^{k} \alpha\right\rangle=\int_{0}^{1} q(\alpha) l n^{k} \alpha d \alpha ; k=1,2$, and $d W(\tau)$ is the Wiener process $\left(\langle d W(\tau)\rangle=0\right.$ and $\left\langle d W(\tau)^{2}\right\rangle=d \tau$, with $\tau$ given by eq. 5$)$. In the present study, parameters are chosen in the following form:

$$
-\langle\ln \alpha\rangle=\left\langle\ln ^{2} \alpha\right\rangle=R e_{+}^{1 / 3}
$$

where $R e_{+}$is the Reynolds number, based on the friction velocity and the channel half-width. The starting condition, $\tau=0$, for this stochastic process (the first grid cell on the wall) is given as follows. We introduce the characteristic value of the frequency $f$ at the wall $f_{+}=\lambda / u_{*}$, where $\lambda$ is determined, as a Taylor-like scale, which can be estimated by the Kolmogorov's scaling in the framework of definitions of wall parameters. The Reynolds number, based on friction velocity, is $R e_{+}=u_{*} h / \nu=h / y_{0} \approx R e_{h}^{3 / 4}$ where $y_{0}$ is the thickness of the viscous layer, and $R e_{h}$ is the Reynolds number based on the center-line 
velocity. One then yields: $\lambda \approx h R e_{h}^{-1 / 2} \approx h R e_{+}^{-2 / 3}$. Similar to KolmogorovOboukhov 62 , the starting condition for the random path given by eq. 6 is sampled from the stationary log-normal distribution of $f / f_{+}$:

$$
P_{0}\left(f / f_{+}\right)=\frac{f_{+}}{f \sqrt{2 \pi \sigma^{2}}} e^{-\frac{\left(\ln \left(f / f_{+}\right)-\mu\right)^{2}}{2 \sigma^{2}}}
$$

with parameters $\sigma^{2}=\ln 2$ and $\mu=-\frac{1}{2} \sigma^{2}$, such that $\langle f\rangle=\left(\left\langle f^{2}\right\rangle-\langle f\rangle^{2}\right)^{1 / 2}=$ $f_{+}$. The stochastic process giving by eq. 6 with initial condition from eq. 8 will relax $f$ from a log-normal distribution at the wall $(\tau=0)$ to the power distribution as the distance to the wall increases $(\tau \rightarrow \infty)$. The evolution through the channel, for distributions of the frequency predicted by the stochastic equation can be compared with the evolution of the frequency computed from DNS, via eq. 4. According to fig. 1a SSAM ensures a good relaxation of the frequency, as the distance to the wall increases.
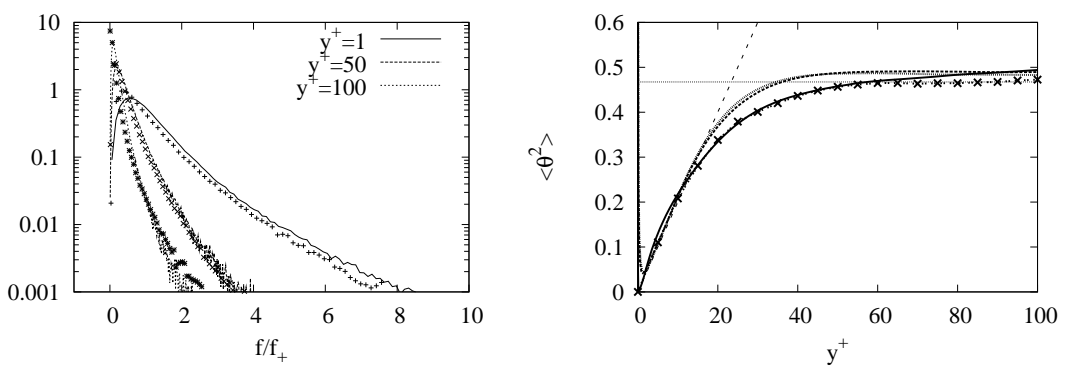

Fig. 1. (a) Distribution of $f / f_{+}$from SSAM (cross) and comparison with DNS (line) at $R e_{+}=590$, for several distances from the wall. (b) Variance of $\theta$ for small scale acceleration from DNS (line) for $R e_{+}=180,590$ and 1000 and from SSAM (cross). Straight line denote the variance of $\theta$ for an isotropic orientation.

In order to emulate the orientation vector of the subgrid scale acceleration, $e_{i}$, we consider a random walk evolving on the surface of a sphere of unity radius. The orientation vector may be defined by two angles which are longitude $\phi$ and latitude $\theta$ :

$$
\left\{\begin{array}{l}
e_{x}=\cos (\theta) \cos (\phi) \\
e_{y}=\sin (\theta) \\
e_{z}=\cos (\theta) \sin (\phi)
\end{array}\right.
$$

The $\phi$ angle characterizes the direction in the streamwise-spanwise $(x, z)$ plan, and the other one, $\theta$, defines the orientation in relation to the normal to 


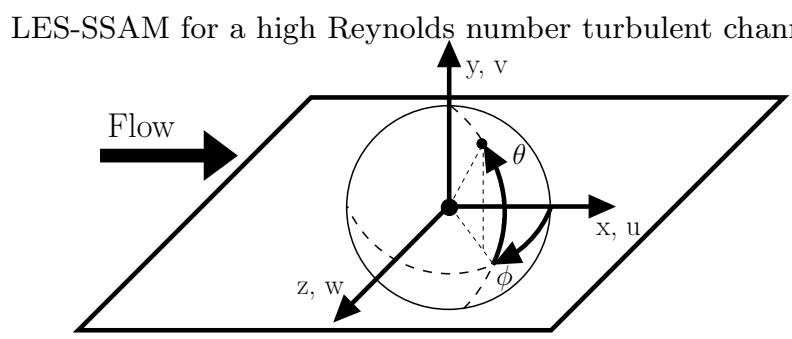

Fig. 2. Coordinate system.

wall direction $(\theta=0$ means acceleration is parallel to the wall, and $\theta= \pm \pi / 2$ means acceleration is normal to the wall), as can be seen on fig 2 . First the computation of $e_{i}$ from DNS was performed. The result are shown in fig. 1b. It is seen that $e_{i}$ relaxes toward isotropy with increasing distance from the wall. Note that in the case of full isotropy, the distributions of $\theta$ and $\phi$ are respectively given by $P_{\text {isotropic }}(\theta)=\cos (\theta) / 2$ and $P_{\text {isotropic }}(\phi)=\pi / 2$.

In order to represent this tendency towards isotropy, we implement a Brownian motion on the sphere. This motion will defines the evolution of the unit vector $e_{i}$. Each increment de of the random walk is given by

$$
d \mathbf{e}=\zeta \mathbf{e} \times d \mathbf{W}\left(d y^{+}\right)
$$

where $d \mathbf{W}\left(y^{+}\right)$is a 3-D Wiener process $\left(\left\langle d \mathbf{W}\left(y^{+}\right)\right\rangle=0\right.$ and $\left\langle d \mathbf{W}\left(y^{+}\right)^{2}\right\rangle=$ $d y^{+}$, with $y^{+}$the distance to the wall in wall unit) and $\times$denotes the vector product. The constant $\zeta$ is given to be consitant with the evolution of orientation vector computed from DNS. We choose $\zeta=25$ which is the characterisic thickness (in wall unit) of the layer near the wall where the orientation is strongly anisotropic. As the distance to the wall increases the random walk covers all the surface of the sphere, ensuring the relaxation towards isotropy for the subgrid scale acceleration orientation. This process is initialized on the wall with:

$$
\left\{\begin{array}{l}
P_{\theta}\left(\theta, y^{+}=0\right)=\delta(\theta) \\
P_{\phi}\left(\phi, y^{+}=0\right)=1 / 2 \pi \quad \text { if } 0 \leq \phi<2 \pi
\end{array}\right.
$$

where $P_{\theta}$ and $P_{\phi}$ are the distribution of $\theta$ and $\phi$ respectively, and $\delta$ is the Dirac distribution, i.e. the orientation vector at the wall is parallel to the wall. The initial condition 11 is coherent with DNS. On fig. 1b we present the evolution of the variance of $\theta$ given by eq. 9 , eq. 10 and eq. 11 and the one computed from the small-scale acceleration of DNS. Good agreement with the DNS is achieved.

\section{Numerical Results and discussion}

In order to make a posteriori tests of this subgrid scale model for acceleration we ran simulations of a pressure driven turbulent channel flow for three 
Table 1. Summery of parameters used for numerical simulations

\begin{tabular}{ccccccc}
\hline \hline Name & $R e_{+}$ & $R e_{c}$ & $N_{x} \times N_{y} \times N_{z}$ & $\Delta x^{+} \times \Delta y^{+} \times \Delta z^{+}$ & $C_{s}$ & $A / h$ \\
\hline DNS & 587 & 12490 & $384 \times 257 \times 384$ & $7.2 \times(0.04 \sim 7.2) \times 3.6$ & - & - \\
DNS [13] & 587 & 12547 & $384 \times 257 \times 384$ & $9.7 \times(0.04 \sim 7.2) \times 4.8$ & - & - \\
LES & 587 & 14160 & $64 \times 65 \times 64$ & $87 \times(0.71 \sim 29) \times 29$ & 0.16 & 0.015 \\
LES-SSAM & 587 & 12760 & $64 \times 65 \times 64$ & $87 \times(0.71 \sim 29) \times 29$ & 0.16 & 0.015 \\
\hline DNS & 1000 & 22250 & $512 \times 385 \times 512$ & $8.2 \times(0.03 \sim 8.3) \times 4.1$ & - & - \\
DNS [5] & 934 & 20960 & $3072 \times 385 \times 2304$ & $7.6 \times(0.06 \sim 7.6) \times 3.8$ & - & - \\
LES & 1000 & 25430 & $96 \times 97 \times 96$ & $99 \times(0.53 \sim 33) \times 33$ & 0.16 & 0.009 \\
LES-SSAM & 1000 & 23380 & $96 \times 97 \times 96$ & $99 \times(0.53 \sim 33) \times 33$ & 0.16 & 0.009 \\
LES & 1000 & 25500 & $64 \times 65 \times 64$ & $147 \times(1.2 \sim 49) \times 49$ & 0.2 & 0.015 \\
LES-SSAM 1000 & 23700 & $64 \times 65 \times 64$ & $147 \times(1.2 \sim 49) \times 49$ & 0.2 & 0.015 \\
\hline DNS [5] & 2003 & 48680 & $6144 \times 633 \times 4608$ & $8.2 \times 8.9 \times 4.1$ & - & - \\
LES & 2000 & 49350 & $128 \times 129 \times 128$ & $147 \times(0.60 \sim 49) \times 49$ & 0.16 & 0.006 \\
LES-SSAM & 2000 & 48950 & $128 \times 129 \times 128$ & $147 \times(0.60 \sim 49) \times 49$ & 0.16 & 0.006 \\
LES & 2000 & 52640 & $64 \times 65 \times 64$ & $295 \times(2.4 \sim 98) \times 98$ & 0.2 & 0.015 \\
LES-SSAM 2000 & 49050 & $64 \times 65 \times 64$ & $295 \times(2.4 \sim 98) \times 98$ & 0.2 & 0.015 \\
\hline \hline
\end{tabular}

Reynolds numbers: $R e_{+}=590,1000$ et 2000 . We used a pseudo-spectral method with integration in time by the explicit Adam-Basforth algorithm for convective terms, and by semi-implicit algorithm for diffusion terms. Further details about the computational code can be found in [2].

The results of LES-SSAM tests have been compared with standard LES and DNS. We used our own DNS data as well as the DNS data from Moser et al. [13] and from Hoyas and Jiménez [5]. For LES and LES-SSAM simulations the classical Smagorinsky model with a wall damping function for the turbulent viscosity has been applied [16]:

$$
\begin{aligned}
\nu_{\text {turb }} & =\left(C_{s} \Delta f_{V D}\right)^{2}|S| \\
|S| & =\left(2 S_{i j} S_{i j}\right)^{1 / 2} \\
f_{V D} & =1-e^{-y / A}
\end{aligned}
$$

with $C_{s}$ the Smagorinsky constant, $\Delta=(\Delta x \times \Delta y \times \Delta z)^{1 / 3}$ the typical cell size, $S_{i j}=\frac{1}{2}\left(\frac{\partial \bar{u}_{i}}{\partial x_{j}}+\frac{\partial \bar{u}_{j}}{\partial x_{i}}\right)$ the resolved rate of strain tensor, $f_{V D}$ the Van Driest function and $A$ the constant controlling the damping of $f_{V D}$. The constant $A$ is computed in order to fulfill the suggestion of Shur et al. [17] for the subgrid length-scale $\ell$ definition: $\ell=\min (y, \Delta), y$ is the distance to the nearest wall. We choose $A$ such that $\Delta f_{V D} \sim \min (y, \Delta)$ by least square regression. The parameters used for these simulations are summarized in table 1.

Note that in this code, Reynolds number are imposed via the setting of $\nu$ and $-\frac{1}{\rho} \frac{\partial P}{\partial x_{i}}$ (the mean pressure gradient). One may use Dean's correlation (Dean, 1978): $\nu=0.110 U_{c} h R e_{+}^{-1.1296}$ and $-\frac{1}{\rho} \frac{\partial P}{\partial x_{i}}=R e_{+}^{2} \nu^{2} / h^{3}$, with $U_{c}$ the center-line velocity, to choose suitable values. As shown in table 1 , the 
Reynolds numbers computed from LES-SSAM are closer to the DNS than the ones computed by LES. For a given set of parameters $\left(\nu\right.$ and $\left.-\frac{1}{\rho} \frac{\partial P}{\partial x_{i}}\right)$, LESSSAM improves both center-line velocity and mass flow rate estimations.

For simplicity reasons, in the following, we only present results from LESSSAM and LES with a $64 \times 65 \times 64$ grid for the three Reynolds numbers. It should be noted that for finer resolutions the differences between LES-SSAM and standard LES are less pronounced, but still present.

Fig. 3a shows evolution of the mean velocity across the channel. As pointed out in table 1 it is clear that LES-SSAM improves mean flow rate estimation as well as center-line velocity prediction. Moreover the mean velocity profile follows the logarithmic law contrary to LES.

On fig. 3 profiles of the standard deviation for streamwise, spanwise and normal to wall velocities are also presented. Standard deviations of streamwise velocity are notably improved. The peak position obtained by LES-SSAM is closer to the DNS than the one obtained with LES. For the spanwise velocity standard deviation the improvement is less visible. However, note that the shape of the profile obtained by LES-SSAM is closer to the DNS one, even if it is overestimated. Finally, the normal to the wall velocity standard deviation is slightly improved in comparison with LES.

Fig. 4a illustrates the computation of turbulent and viscous stresses, $\tau_{\text {turb }}=-\rho\left\langle u^{\prime} v^{\prime}\right\rangle$ and $\tau_{\text {visc }}=-\rho \nu\left\langle\frac{\partial u}{\partial y}\right\rangle$, respectively $(\langle$.$\rangle denotes ensem-$ ble average $)$. The results are presented as ratios $\tau_{\text {turb }} /\left(\tau_{\text {turb }}+\tau_{\text {visc }}\right)$ and $\tau_{\text {visc }} /\left(\tau_{\text {turb }}+\tau_{\text {visc }}\right)$. Here again the advantage of the LES-SSAM approach versus the classical LES is explicitly seen, and can be interpreted as a better estimation of momentum fluxes in the normal to the wall direction. In addition to this, from fig. $4 \mathrm{a}$ it can be seen that close to the wall the viscous stress is dominant, whereas as we move away from the wall the turbulent stress becomes preponderant. The LES-SSAM approach enables improvement of both the viscous and the turbulent stress away and close to the wall.

Velocity spectra are shown on fig. 4b. From this figure we can see that the anomalous small scale (high wave number) damping inherent to LES can be reduced by LES-SSAM. Fig. 4c represents the evolution of the longitudinal autocorrelation coefficient for the streamwise velocity component along the channel. Improvement of the decorrelation length can be seen, indicating that integral length scales, and so the size of strucutres in the near wall, computed by LES-SSAM are closer to DNS than in the classical LES.

This result is mainly due to the fact that decorrelation is ensured by small scale fluctuations modelled with LESS-SSAM as seen by the spectra on fig. $4 \mathrm{~b}$.

Fig. $4 \mathrm{~d}$ illustrates the distribution of the spanwise acceleration. The distributions obtained by DNS present stretched tails, as a manifestation of intermittency. From fig. 4d it can be seen that in agreement with the DNS, the distributions of the $a_{z}$, obtained by LES-SSAM, present the stretched tails, while these distributions computed by LES stay close to the Gaussian distribution. By introducing the LES-SSAM model, small scale intermittency is included in the modelling of the acceleration. 

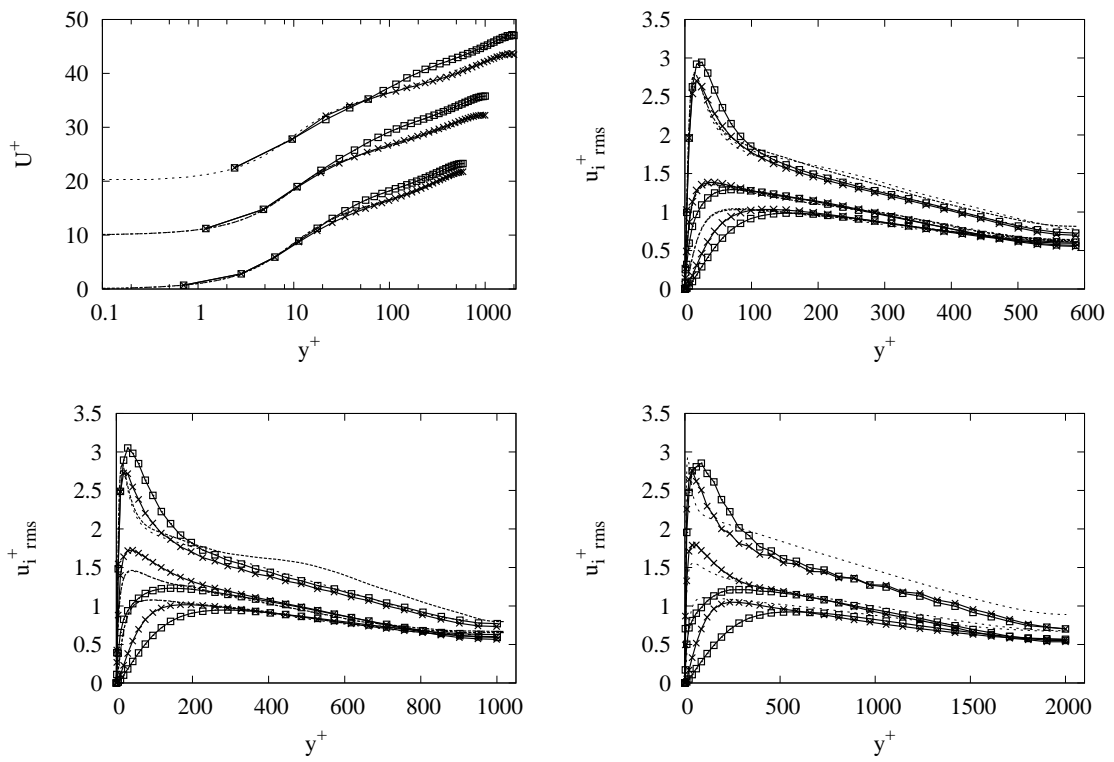

Fig. 3. (a) Streamwise mean velocity for $R e_{+}=590, R e_{+}=1000$ and $R e_{+}=$ 2000 from bottom to top, respectively, shifted by 10 wall units upward. Standard deviation of streamwise $(u)$, spanwise $(w)$ and normal to the wall $(v)$ velocity, for (b) $R e_{+}=590$, (c) $R e_{+}=1000$ and (d) $R e_{+}=2000$ Square: LES; cross: LESSSAM; dash: DNS (only for $R e_{+}=590$ and $R e_{+}=1000$ ); dots: DNS from [13] for $R e_{+}=590$ and from [5] for $R e_{+}=1000$ and $R e_{+}=2000$.

\section{Conclusion}

In the framework of the LES-SSAM approach, a new SGS model is proposed for the subfilter scale acceleration in order to include the intermittency effects in the near-wall region of a high-Reynolds number channel flow. In this model, the modulus and the orientation of the non resolved acceleration are considered as two independent stochastic variables. The modulus is a function of a subgrid frequency given by a fragmentation stochastic process under the scaling symmetry. The mean and standard deviation of the process are functions of the Reynolds number. For the orientation of the acceleration a random walk evolving on the surface of a sphere is proposed. The orientation of the acceleration is such that away from the wall the process relaxes towarss isotropy. This was chosen in agreement with the DNS.

The results for the model proposed here are compared with DNS data $\left(R e_{+}=590,1000\right.$ and 2000) and standard LES. The comparisons show that mass flow rate estimations and mean velocity profiles are improved by introducing the LES-SSAM model. In addition to this a better prediction near the wall of the streamwise standard deviation, the viscous and turbulent stresses 

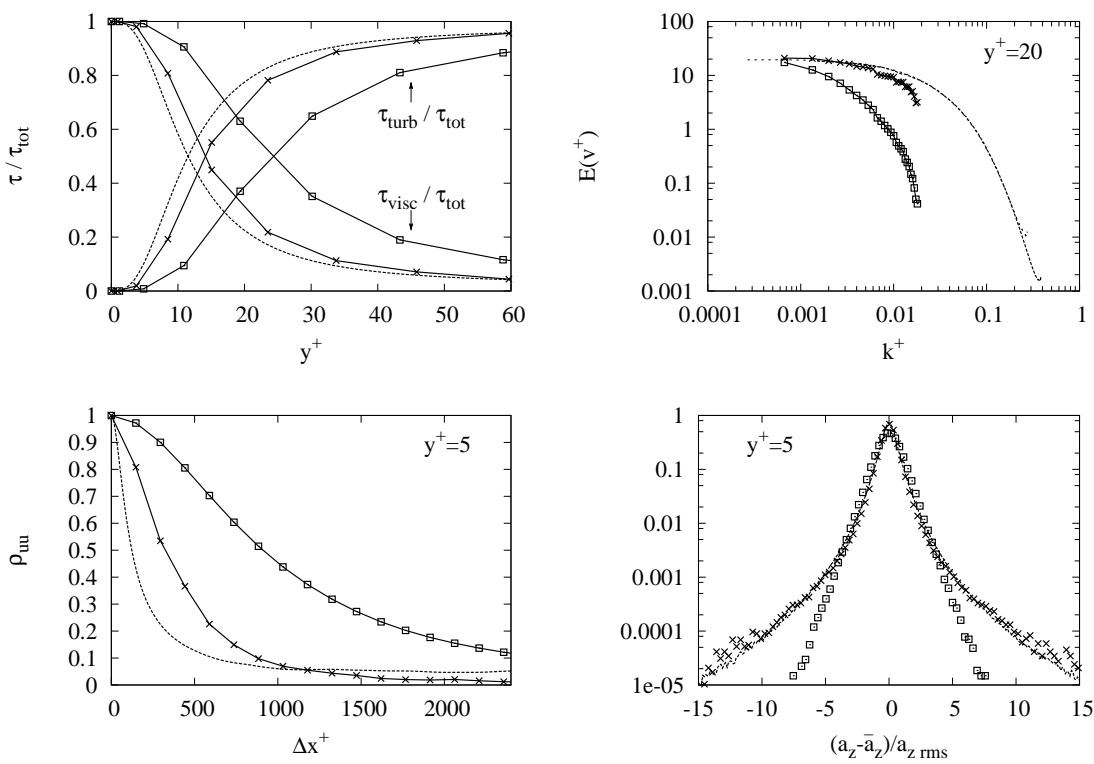

Fig. 4. (a) Fractions of turbulent $\tau_{t u r b}=-\rho\left\langle u^{\prime} v^{\prime}\right\rangle$ and viscous $\tau_{v i s c}=-\rho \nu\left\langle\frac{\partial u}{\partial y}\right\rangle$ stresses compared to the total one $\tau_{\text {tot }}=\tau_{\text {visc }}+\tau_{\text {turb }}$. (b) Normalized longitudinal $1-\mathrm{D}$ spectra of normal to wall velocity for $y^{+}=20$. (c) Longitudinal autocorrelation of streamwise velocity at $y^{+}=5$. (d) Distribution of spanwise acceleration at $y^{+}=$ 5. $R e_{+}=1000$. Square: LES; cross: LES-SSAM; dash: DNS; dots: DNS from [5] (uniquely for (b)).

are obtained with LES-SSAM. Small scale dumping inherent to LES is avoided with LES-SSAM. Finally, improved longitudinal velocity decorelation is obtained as well as the prediction of the stretched tails in the acceleration distribution.

Acknowledgement. M. Buffat is acknowledged for the development of the computational code. The authors express their gratitude to F. Laadhari who kindly provided his DNS fields. This work was granted access to the HPC resources of CINES under the allocation 2009-c200902560 made by GENCI (Grand Equipement National de Calcul Intensif)

\section{References}

1. R. J. Adrian, C. D. Meinhart, and C. D. Tomkins. Vortex organization in the outer region of the turbulent boundary layer. Journal of Fluid Mechanics, 422:1-54, 2000. 
2. M. Buffat, L. Le Penven, and A. Cadiou. An efficient spectral projection method on divergence-free subspaces for transition analysis in wall bounded flow. Journal of computational physics, submitted, 2009.

3. J. A. Domaradzki and N. A. Adams. Direct modelling of subgrid scales of turbulence in large eddy simulations. Journal of Turbulence, 3:0-24, 2002.

4. M. Gorokhovski and V. L. Saveliev. Statistical universalities in fragmentation under scaling symmetry with a constant frequency of fragmentation. Journal of Physics D: Applied Physics, 41:085405, 2008.

5. S. Hoyas and J. Jiménez. Reynolds number effects on the reynolds-stress budgets in turbulent channels. Physics of Fluids, 20(10):101511, 2008.

6. N. Hutchins and I. Marusic. Evidence of very long meandering features in the logarithmic region of turbulent boundary layers. Journal of Fluid Mechanics, 579:1-28, 2007.

7. J. Jiménez, J. C. Del Alamo, and O. Flores. The large-scale dynamics of nearwall turbulence. Journal of Fluid Mechanics, 505:179-199, 2004.

8. D. Kaftori, G. Hetsroni, and S. Banerjee. Funnel-shaped vortical structures inwall structures. Physics of Fluids, 6(9):3035-3050, 1994.

9. C. Lee, K. Yeo, and J.-I. Choi. Intermittent nature of acceleration in near-wall turbulence. Physical Review Letters, 92(14), 2004.

10. C. Meneveau and J. Katz. Scale-invariance and turbulence models for largeeddy simulation. Annual Reviews of Fluid Mechanics, 32:1-32, 2000.

11. N. Mordant, J. Delour, E. Léveque, A. Arnéodo, and J.-F. Pinton. Long time correlations in lagrangian dynamics: a key to intermittency in turbulence. Pysical Review Letters, 89(25), 2002.

12. N. Mordant, E. Lévêque, and J.-F. Pinton. Experimental and numerical study of the Lagrangian dynamics of high Reynolds turbulence. New Journal of Physics, 6:116, 2004.

13. R. D. Moser, J. Kim, and N. N. Mansour. Direct numerical simulation of turbulent channel flow up to $R e_{\tau}=590$. Physics of Fluids, 11(4):943-945, 1999.

14. N. Park and K. Mahesh. A velocity-estimation subgrid model constrained by subgrid scale dissipation. Journal of Computational Physics, 227:4190-4206, 2008.

15. V. Sabelnikov, A. Chtab, and M. Gorokhovski. The coupled LES - sub-grid stochastic acceleration model (LES-SSAM) of a high Reynolds number flows. In Advances in Turbulence XI, volume 117, pages 209-211, 11th EUROMECH European Turbulence Conference, June 25-28, 2007, Porto, Portugal, 2007. Springer Proceedings in Physics.

16. P. Sagault. Large Eddy Simulation for Incompressible Flows: An introduction. Springer Verlag, second edition, 2002.

17. M. L. Shur, P. R. Spalart, M. Kh. Strelets, and A. K. Travin. A hybrid RANSLES approach with delayed-DES and wall-modelled LES capabilities. International Journal of Heat and Fluid Flow, 29(6):1638-1649, 2008.

18. S. Toh and T. Itano. Interaction between a large-scale structure and near-wall structures in channel flow. Journal of Fluid Mechanics, 524:249-262, 2005.

19. C. D. Tomkins and R. J. Adrian. Spanwise structure and scale growth in turbulent boundary layers. Journal of Fluid Mechanics, 490:37-74, 2003. 